\title{
HISTORIA
}

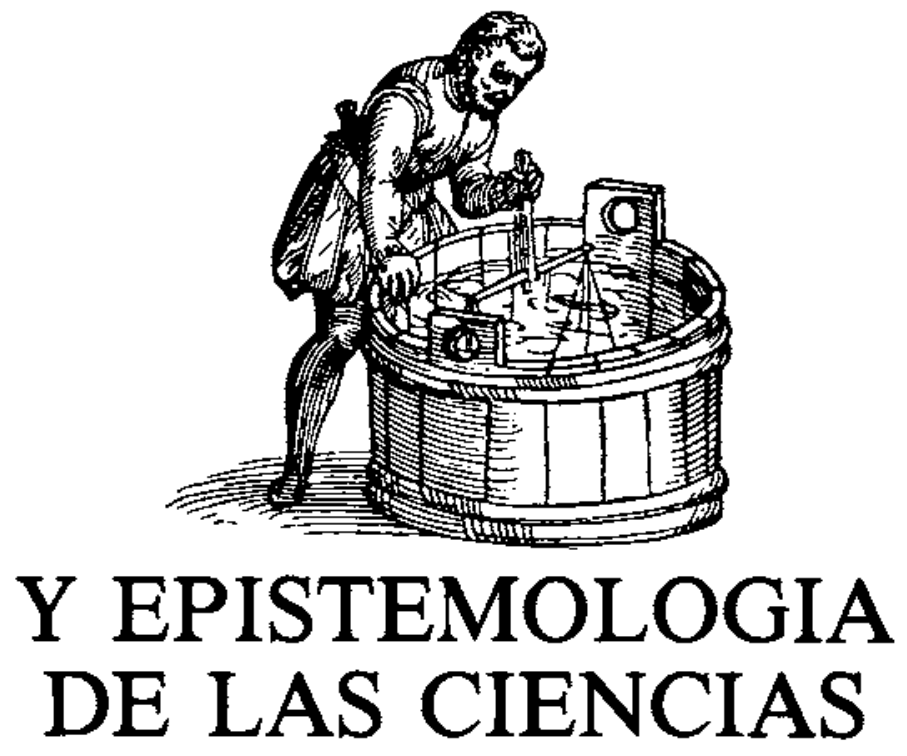

\section{EL PAPEL DEL PÉNDULO EN LA CONSTRUCCIÓN DEL PARADIGMA NEWTONIANO}

SOLAZ PORTOLÉS, J.J.' y SANJOSÉ LÓPEZ, V2.

1. Institut de Batxillerat de Cullera.

2. Departament de Didàctica de les Ciències Experimentals. Universitat de València. Apartado de Correos 22045. 46071 València.

\section{SUMMARY}

This paper shows the important role of one of the most common instruments, the pendulum, in the construction of the newtonian paradigm. From the analysis of some interesting applications carried out by the greatest scientists of that time, we conclude that the appropriate use of the pendulum in the classroom can be an easy way to introduce historical and epistemological evolution of basic physical concepts.

\section{INTRODUCCIÓN}

Desde que se ha establecido la conexión entre las aportaciones de la Historia y Epistemología de la Ciencia y Ia Didáctica de las Ciencias, muchos han sido los trabajos que muestran las aplicaciones de aquéllas en la labor docente (Matthews 1990). El paralelismo aparente entre el desarrollo de las ideas históricas y de las concepciones científicas de los aprendices (Saltiel y Viennot 1985) ha permitido el reencuentro entre las dos áreas de conocimiento y, con ello, su mutuo enriquecimiento.
En esta línea, el trabajo que se presenta aquí trata de poner de relieve la notable influencia que los estudios con uno de los instrumentos más humildes y presentes en nuestros laboratorios, el péndulo, han ejercido en el desarrollo conceptual de la ciencia mecánica desde su principio, contribuyendo a conformar uno de los pilares de la física clásica: el paradigma newtoniano. Por otro lado, se pretende evidenciar algunas consecuencias que pueden derivarse de dichos estudios y que resultan ser de 
gran interés diđáctico y espistemológico. Puesto que el péndulo ha sido a la vez, testigo y motor de la construcción de la mecánica desde el siglo XVI al XIX, eI análisis de su contribución al establecimiento de principios y leyes fundamentales, permite su uso didáctico como hilo conductor de una investigación dirigida en el aula, y puede acercar a nuestros alumnos al trabajo de algunos de los «padres» de la física clásica.

Para hacernos una idea de la importancia instrumental que tuvo el péndulo, basta con señalar que el número de referencias bibliográficas sobre su teoría y aplicaciones citadas en Bibliographie du pendule por Wolf (1889b), asciende aproximadamente a unas mil doscientas en el período comprendido entre 1632 -año de publicación en Florencia de la obra de Galiteo Dialogo intorno ai due massimi sistemi del mondo, Tolemaico e Copernicanoy 1885 .

Indicaremos en relación con estos trabajos publicados, que el crecimiento mayor de su número se produjo tras la publicación por Foucault en 1851 de Demonstration physique du mouvement de rotation de la Terre au moyen du pendule, y que en ellos aparecen cientificos tan sobresalientes como Galileo, Huygens, Newton, Hooke, Halley, Mariotte, Bernouilli, Celsius, D'Alambert, Poisson, Young, Sabine, Stokes, etc.

\section{GALILEO: LA CAÍDA DE GRAVES Y EL PENDULO}

Parece claro que desde mucho antes del nacimiento de Galileo muchas personas habían visto oscilar un objeto del extremo de una cuerda hasta que fínalmente quedaba en reposo. El análisis que se hizo de su movimiento por parte de los aristotélicos fue el de una caída dificultosa de un cuerpo pesado unido a un hilo, que sólo podía quedar parado en su posición más baja después de un tiempo considerable. Galileo vio el péndulo desde otra perspectiva y rompió con el paradigma aristotélico consiguiendo de este modo «descubrir» el péndulo: un cuerpo que seguía un movimiento periódico (Khun 1987a). Tras esta ruptura, la atención de Galileo se orientó hacia la forma circular del movimiento $\mathrm{y}$, según creía, su isocronismo, llevándole a formular en la Jornada Primera de los Discorsi la ley del isocronismo pendular bajo la forma de una proporcionalidad entre el «tiempo de vibración» y la raíz cuadrada de la longitud del péndulo (Azcárate et al. 1988).

No obstante, lo que nos parece más interesante son las consecuencias dinámicas extraídas de sus experimentos con el péndulo, sin entrar en la controversia mantenida por los historiadores de la ciencia «empiristas» y «racionalistas" acerca de la importancia real de la experimentación en los descubrimientos de Galileo (Thuillier 1983 y 1990).

Así, observando las equivalencias entre el movimiento de una bola que rueda por un plano inclinado y el movimiento pendular (Khun 1987a) Galileo extrajo sólidos argumentos para:

a) Entrever, sin Ilegar a enunciar ninguna ley, la noción de inercia, principio según el cual los cuerpos tienden a conservar su estado de reposo o de movimiento (Crombie 1987, Koyré 1980).

b) Confirmar la hipótesis de que las velocidades adquiriđas por un cuerpo que baja por planos de diferentes inclinaciones son iguales cuando las atturas de los planos son iguales. Con este fin realizó el experiemento del péndulo y el clavo; en él el movimiento del péndulo se ve alterado al chocar la cuerda con un clavo, y la masa pendular alcanza siempre la misma altura independientemente de la posición del clavoe incluso de su participación (Fig. 1) (Losee 1987).

Figura 1

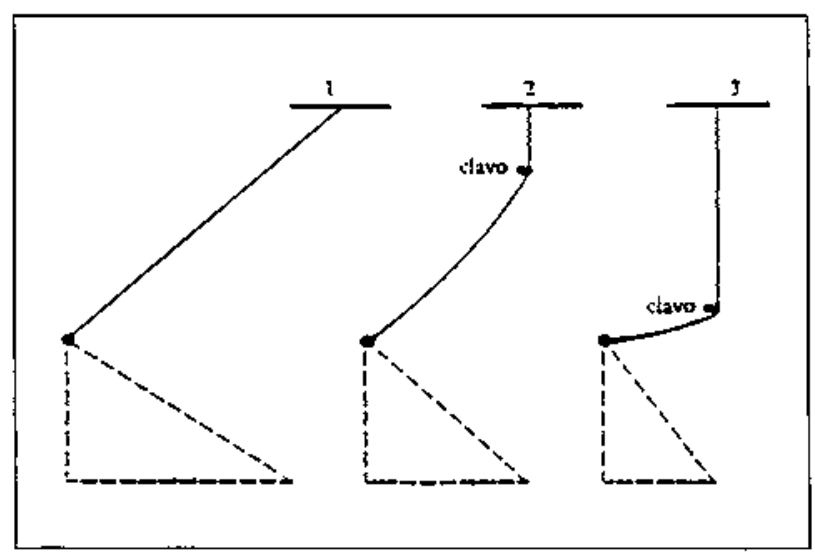

c) Reconocer que los tiempos de caída de los graves son independientes de sus pesos, en contra de lo que sostenía la física peripatética (Khun 1987a, Papp 1945). Es importante señalar al respecto que, para Galileo, el peso era todavía una tendencia intrínseca hacia abajo, no algo que dependía de una relación extrínseca con otro cuerpo atrayente, como generalizara Newton en su Teoría de la Gravitación Universal (Crombie 1987).

\section{HUYGENS Y EL PÉNDULO: \A MEDICIÓN DEL TIEMPO, LA FUERZA CENTRIFUGA Y LA CONSERVACIÓN DE LA ENERGÍA}

Como apunta Koyré (1983), la ciencia moderna en el momento de su nacimiento se encontraba en una situación paradójica: poseía leyes matemáticas exactas para la dinámica y a su vez era imposible aplicarlas porque no se podían efectuar mediciones precisas de una magnitud fundamental como es el tiempo.

Huygens era consciente de ello, como también lo era de la necesidad práctica de disponer de relojes precisos, por 
lo que al comienzo de su carrera científica se dedicó a dar solución a este problema. En un estudio sobre el péndulo simple, llevado a cabo en 1659, Huygens compar6 la duración de la «caída» del pérudulo con la de una caída libre. El resultado de tal estudio, publicado en la cuarta parte de su Horologium Oscillatorium, es totalmente equivalente a la formula $\mathrm{T}=2 \pi \sqrt{\mathrm{l} / \mathrm{g}}$. Por supuesto en su deducción usó aproximaciones que descartaban la dependencia del período con la amplitud (Bos 1980). Pero después, comprobó que las oscilaciones de un péndulo simple no son estrictamente isócronas sino que dependen de la amplitud de la oscilación además de la longitud del hilo. Para corregir el discronismo que podía generar errores en las medidas de tiempos, pensó en adaptar unas guías en forma de arcos curvados simétricamente en torno al punto de fijación del hilo que permitieran reducir la longitud del péndulo cuando aumentase la amplitud de las oscilaciones (Dugas y Costabel 1988).

Sin embargo sus inquietudes matemáticas no quedaron satisfechas y su objetivo técnico pasó a ser teórico: deseaba hallar la trayectoria de la lenteja que conducía a un perfecto isocronismo. En 1658 Huygens descubrió que la completa independencia de la amplitud, y en consecuencia el perfecto tautocronismo, podía ser obtenido si el movimiento de la masa pendular describía una cicloide, curva ésta que había estudiado previamente en conexión con un problema matemático planteado por Pascal. Necesitaba entonces encontrar la forma de las guías que regulan la longitud para conseguir oscilaciones cicloidales, y sus investigaciones le llevaron a un hallazgo matemático de gran importancia: la involuta (también llamada evolvente) de una cicloide es otra cicloide igual, o a la inversa, la evoluta de una cicloide es otra cicloide igual a elia. Expresado en otros términos, si suspendemos del punto $O$ (Fig. 2) un péndulo entre dos semiarcos de una cicloide, OA y OC, de igual longitud que éste, entonces $m$ oscilará describiendo un arco de cicliode $\mathrm{ABC}$. Tras la iniciación de la teoría de involutas y evolutas por Huygens se rectificaron otras muchas curvas, poniéndose en cuestión el dogma peripatéticocartesiano de la no rectificación de las curvas algebraicas (Boyer 1986).

Figura 2

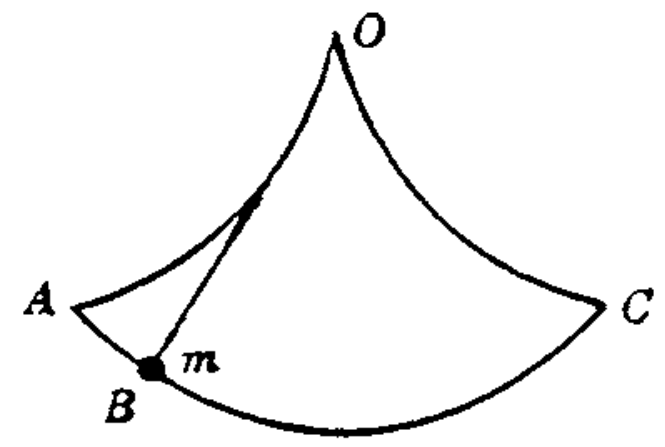

Sin embargo, Huygens fue todavía mucho más lejos al considerar en sus trabajos no solamente el péndulo ideal, matemático, sino que se abocó al problema más complicado del péndulo real, físico. Para ello, modeló la solución al problema basándose en el péndulo puntual galileano: imaginó el péndulo físico compuesto por un conjunto de partículas, cada una de las cuales puede ser considerada como si fuese un péndulo matemático (Khun 1987a).

Centrando de nuevo la atención sobre la duracion de las oscilaciones, introdujo el concepto de centro de oscilación de un péndulo físico, que define a partir de la longitud $\lambda$ de un péndulo simple que oscile isocrónicamente con el compuesto, y que coincide con el punto $O$ (Fig. 3) situado a una distancia $\lambda$ del eje sobre la línea que une $O$ con el centro de gravedad $Z$. Dicho concepto le permitió tratar un péndulo físico como matemático (Papp 1945).

Figura 3

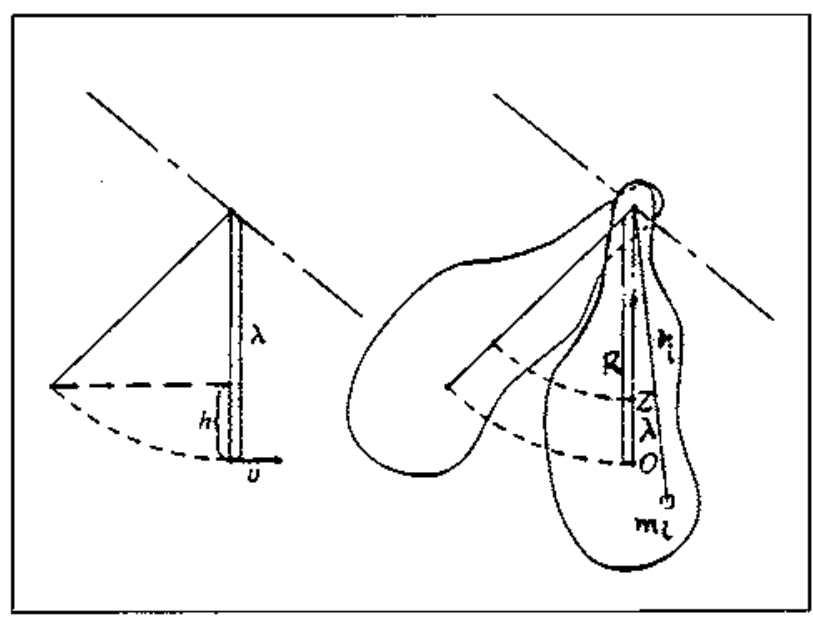

En la determinación de los centros de oscilación de diferentes péndulos, Huygens partió de dos principios:

-El centro de gravedad no puede alcanzar mayor altura que aquélla de donde descendió.

-En ausencia de friccion el centro de gravedad describe arcos iguales durante su ascenso y descenso.

Veamos un razonamiento similar al que Huygens utiliz6 (Bos 1980).

Si consideramos el péndulo compuesto constituido por partículas de masa $m_{j}$, cuyas distancias al eje son $r_{i}$ (Fig. 3), el centro de gravedad $Z$ se encuentra a una distancia $R$ del eje. Llamemos $\lambda$ a la longitud del péndulo simple isócrono cuya pénđola se halla inicialmente a una altura $\mathrm{h}$ sobre la posición más baja, y y a la velocidad de aquélla en ésta. Es evidente que cuando $O$ descienda la distancia $h, Z$ descenderá la distancia $R h / \lambda$. Además cada una de las partículas $\mathrm{m}_{\mathrm{i}}$ cuando el punto $\mathrm{O}$ pase por la posición más baja tendrá una velociđad $v_{i}=r_{i} v / \lambda$ y -según afirman los principios formulados 
por Huygens-alcanzará posteriormente una altura $h_{i}$. Por otra parte, de acuerdo con la ley de Galileo de caf́da de Ios cuerpos $v_{i}{ }^{2}=k h_{i}$ y $v^{2}=k h$, por tanto:

$h_{i}=h\left(v_{i}^{2} / v^{2}\right)=h\left(r_{i}^{2} / \lambda^{2}\right)$

$\mathrm{Y}$ como $\mathrm{Z}$ alcanzará en el ascenso una altura:

$h^{\prime}=\Sigma m_{i} h_{i} / \Sigma m_{i}=\Sigma m_{i} \cdot\left(r_{i}^{2} / \lambda^{2}\right) \cdot h / \Sigma m$

que ha de ser también igual a la descendida, R.h/ $\lambda$, tenemos:

$\mathrm{Rh} / \lambda=\Sigma \mathrm{m}_{1} \cdot\left(\mathrm{r}_{\mathrm{i}}^{2} / \lambda^{2}\right) \cdot \mathrm{h} / \mathrm{M}$

siendo $M$ la masa del péndulo compuesto. Por consiguiente la distancia entre el centro de oscilación $O$ y el punto de suspensión viene dada por la expresión:

$\lambda=\Sigma \mathrm{m}_{\mathrm{i}} \mathrm{r}_{\mathrm{i}}{ }^{2} / \mathrm{M} \cdot \mathrm{R}$

cuyo numerador Euler convino en llamar años después Momento de Inercia (Papp 1945).

De lo expuesto con anterioridad se visIumbra que Huygens fue el verdadero introductor de la Dinámica del Sólido Rígido (Truesdell 1975), y además, con sus principios para el cálculo de centros de oscitación inició el camino que conduciría al Principio de Conservación de la Energía (Khun 1987b).

La contribución de Huygens a la construcción de la Mecánica y, en particular, al estudio del péndulo no acaba aquí. Realizando experimentos sobre el movimiento de péndulos y el movimiento circular en general, y orientado por las lecturas de Descartes, observó la tendencia de los cuerpos a alejarse del centro cuando éstos seguían una «órbita circular que debía compensarse para que girasen equilibradamente (recuérdese que este razonamiento es prenewtoniano). A dicha tendencia la llamó fuerza centrífuga, y sus cálculos la evaluaron como proporcional al cuadrado de la velocidad e inversamente proporcional al radio del círculo (Casadellà y Bibiloni 1985, Mason 1985).

\section{HOOKE, EL PENDULO Y EL ANÁLISIS DEL MOVIMIENTO PLANETARIO}

Como señala Cohen (1981), Hooke dio un paso decisivo hacia la Gravitación Universal al introducir un nuevo método de analizar el movimiento orbital de los planetas. La esencia del método consiste en considerar que el movimiento observado en cada punto es el resultante de dos componentes: una «inercial» y otra «centrípeta». La componente inercial tiende a lanzar el planeta a lo largo de una recta tangente a la curva descrita, mientras que la centrípeta desvía continuamente el planeta de la trayectoria anterior hacia el Sol. En consecuencia, la hipótesis de Hooke supone la existencia de una fuerza que opera entre el Sol y cada uno de los planetas y que desvía a éstos de su movimiento inercial rectilíneo atrayéndolos hacia el centro del Sol.

A pesar de este brillante análisis, Hooke desconocía cómo relacionar la intensidad de una fuerza con la desviación que produce, y por consiguiente, no pudo demostrar la operatividad de su hipótesis. Sin embargo, concretó sus ideas mediante un modelo icónico: un péndulo cónico (Fig. 4). Cuando se separa la lenteja de su posición de equilibrio, la fuerza resultante está dirigida hacia su posición de equilibrio y oscila sobre un plano que contiene la cuerda y la lenteja, pero si recibe un impulso lateral, la lenteja describe una curva similar a la «órbita de un planeta. Según sea el impulso, seguirá un tipo de curva u otra. Así, el péndulo cónico convertía en clara y plausible su hipótesis: una única fuerza centraI puede producir una órbita cerrada (Khun 1985).

Figura 4
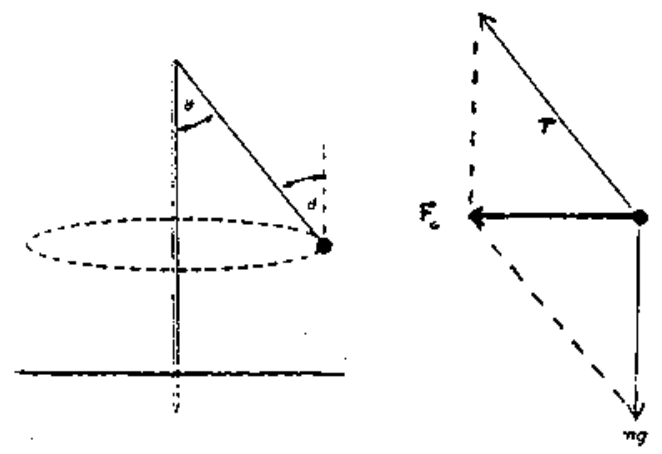

\section{NEWTON Y EL PÉNDULO: MASA, PESO Y TEORÍA DE LA GRAVITACION UNIVER- SAL}

El escabroso problema de interpretación de la idéntica aceleración en la caída para cuerpos distintos por parte de los que, como Galiteo, habían puesto en tela de juicio la cinemática aristotélica, halló finalmente solución con Newton. Éste separó el concepto de peso, motivado por la fuerza externa de la gravedad que actúa sobre el cuerpo, del de masa, que define como la cantidad de materia existente en él (Crombie 1987, Papp 1945). Y todavía mucho más importante es la conclusión de sus experimentos con péndulos de la misma longitud y distintos materiales reflejados en la Proposición XXIV, Teorema XIX, del Libro Segundo de los Principia: la proporcionalidad entre el peso y la masa de un cuerpo (Newton 1987).

También por medio de péndulos, y basándose en las investigaciones efectuadas previamente por Wren, Wallis, Huygens y Mariote (Dugas y Costabel 1988), Newton verificó su tercera Ley del Movimiento, la igualdad de la acción y la reacción (Newton 1987). Esta ley es la más 
original de las tres Leyes del Movimiento, como el mismo Newton reconoce, concediéndole a Galìleo las dos primeras (Dugas y Costabel 1988), y todavía hay quienes la utilizan equivocadamente al asociarla con una supuesta condición de equilibrio y no con una situación de interacción entre dos cuerpos y de simetría en el Universo.

Por último, no podemos dejar de mencionar el papel subsidiario que, a nuestro juicio, desempeñaron los estuđios con y sobre el péndulo en la formulación de la Ley de la Gravitación Universal. En primer lugar, como acabamos de ver, mediante experimentos con péndulos Newton determina que el peso de un cuerpo es directamente proporcional a su masa. En segundo lugar, recordemos que Huygens derivó, merced a sus trabajos con pénđulos, la relación $\mathrm{v}^{2} \mathrm{r}$ para la fuerza centrífuga. En tercer lugar, el péndulo cónico ratificó el análisis del movimiento de los planetas efectuado por Hooke. Además parece ser que con la relación $\mathrm{v}^{2} \mathrm{r}$ y la tercera ley de Kepler, Hooke llegó a sugerirle a Newton que la fuerza centrípeta hacia eI Sol era inversamente proporcional al cuadrado de la distancia, ley ésta que fue crucial en la elaboración por Newton de la Teoría de la Gravitación Universal (Cohen 1981). Y en cuarto lugar, ¿por qué no admitir la posibilidad de que el modelo matemático del que partió Newton en el desarrollo de sus ideas sobre gravitación-una masa puntual moviéndose en torno a un centro de fuerzas-se inspiró en el tratamiento «puntual» que hizo Huygens para el péndulo compuesto?

\section{EL PÉNDULO DE FOUCAULT Y LOS SISTE- MAS DE REFERENCIA INERCIALES O PRI- VILEGIADOS}

Los físicos que continuaron la labor iniciada por Newton en la ciencia mecánica a lo largo del siglo XVIII, e incluso los creadores de la Mecánica Analítica, centraron todos sus esfuerzos en desplegar consecuencias matemáticas de los principios establecidos para el movimiento, y obviaron la reflexión acerca deI vital papel que en el estudio del movimiento tiene el tipo de sistema de referencia (TruesdeI1 1975).

En este contexto, el físico francés León Foucault publicó en 1851 una memoria en la que se recogen sus experiencias con un péndulo de $28 \mathrm{~kg}$ suspendido de un alambre de acero de $67 \mathrm{~m}$ en la cúpula del Panteón de los Inválidos de París. En ella, partiendo de la premisa de que el planođe oscilación del péndulo permanece invariable, probó la rotación de la Tierra sobre sí misma mediante la evidencia experimental de la rotación del plano de oscilación respecto de la Tierra (Wolf 1889a).

Destacaremos que, muy probablemente, Foucault no conocía las investigaciones llevadas a cabo por Coriolis con anterioridad y en las que realizaba modificaciones en la ley fundamental de la Dinámica para el movimiento relativo de un cuerpo respecto de un sistema de referencia dotado, a su vez, de un movimiento respecto de la
Tierra; e incluso, no comprendió la trascendencia de sus resultados para la mecánica. La interpretación de los resultados obtenidos por ambos físicos años más tarde, puso el acento en la noción de sistema de referencia privilegiado -sistema de referencia inercial-y reconoció que las leyes del movimiento enunciadas por Newton llevan en sí mismas el postulado de la existencia de sistemas de referencia privilegiados para el movimiento (Costabel 1988).

De este modo, el descubrimiento hecho con un péndulo, el de Foucault, asentó más si cabe el paradigma newtoniano, ayudando a resolver una de las dificultades más grandes de dicho paradigma y que el mismo Newton advirtió: explicitas el sistema coordenado respecto del cual se cumplen las leyes del movimiento (Symon 1979). Por otra parte, ello permitió algunos años después que se buscara experimentalmente una evidencia de la existencia de tales sistemas privilegiados, y con el «fracaso» consiguiente, el advenimiento del paradigma relativista.

\section{CONCLUSIONES}

I. En el presente trabajo hemos visto cóno los científicos, para resolver problemas, recurren a las soluciones dadas para otros problemas. Así, Galileo asimila la caída por un plano inclinado al movimiento de la lenteja de un péndulo; Huygens al introducir el concepto de centro de oscilación de un péndulo físico reduce éste a un conjunto de péndulos puntuales galileanos; Hooke encuentra en eI péndulo cónico el modelo apropiado para el estudio del movimiento de los planetas alrededor del Sol; y Newton, al que muy probablemente el modelo de Huygens para el péndulo compuesto junto con los trabajos de Hooke avivaron su genio para formular la Ley de la Gravitación Universal. Con ello, podemos mostrar a nuestros alumnos una de las fases claves en la construcción del pensamiento científico: la modelización de la realidad natural (Matthews 1990).

II. Se nos revela el péndulo como un instrumento decisivo en la construcción de la mecánica newtoniana, pues como hemos visto participó en :

a) La elaboración de las leyes del movimiento, especialmente en la de inercia y acción-reacción.

b) EI establecimiento de la Ley de la Gravitación Universal, al permitir el descubrimiento de que el peso es proporcional a la masa, el cálculo đe la relación $\mathrm{v}^{2} / \mathrm{r}$ para la fuerza centrípeta, la confirmación del movimiento planetario por la acción de una fuerza central, y sugerirle quizá a Newton el modelo matemático de una masa puntual sometida a una fuerza central y su justificación.

c) El hallazgo del principio de conservación de la energía mecánica.

d) La iniciación de Ia Dinámica del Sólido Rígido. 
e) La concesión de la atención necesaria a los sistemas de referencia inerciales y el establecimiento de los límites de validez de las leyes newtonianas.

III. Gracias al péndulo, las «leyes» inconexas del movimiento que enunciaron Galileo y Huygens, fueron sintetizadas y derivadas por la teoría newtoniana de la gravitación y del movimiento. Para ello Newton en su teoría formuló con términos teoréticos más precisos dichas leyes e introdujo hipótesis nuevas que establecieron vínculos lógicos entre aquéllas (Bunge 1985, Hempel 1987).

IV. Como han demostrado algunos didactas de la Física (Driver 1986, Gil 1986), los conceptos y principios del paradigma newtoniano no suelen ser asimilados a causa de la existencia en los alumnos de esquemas conceptuales propios que son difícilmente sustituidos por los modelos de enseñanza/aprendizaje tradicionales. Por añadidura, tales esquemas conceptuales parecen guardar un cierto

\section{REFERENCIAS BIBLIOGRÁFICAS}

AZCÁRATE, C., GARCÍA DONCEL, M. y ROMO J., 1988. Galileo Galilei: La nueva ciencia del movimiento. (UAB y UPC: Bellaterra).

BOS, H.J.M., 1980. Christian Huygens, en Dictionary of Scientific Biography. (New York Scribners: New York).

BOYER, C.B.,1986. Historia de la Matemática.(Alianza:Madrid).

BUNGE, M., 1985. La investigación científica. (Ariel: Barcelona).

CASADELLÀ, I. y BIBILONI, L., 1985. La construcción histórica del concepto de fuerza centrípeta en relación con las dificultades de su aprendizaje, Enseñanza de las Ciencias, Vol 3, pp. 217-224.

COHEN, I.B., 1981, EI descubrimiento newtoniano de Ia gravitación, Investigación y Ciencia, Mayo, pp. 111-120.

COSTABEL, P., 1988. Apogeo e incertidumbres de la Mecánica Clásica, en Historia General de las Ciencias. (Orbis: Barcelona).

CROMBIE, A.C., 1987. Historia de la ciencia. De San Agustín a Galileo. (Alianza: Madrid).

DRIVER, R., 1986. Psicología cognoscitiva y esquemas conceptuales de los alumnos, Enseñanza de las Ciencias, Vol 4, pp. 3-15.

DUGAS, R. y COSTABEL, P., 1988. Nacimiento de una nueva ciencia: La Mecánica, en Historia General de las Ciencias. (Orbis: Barcelona).

GIL, D., 1986. La metodología cientifica y la enseñanza de las ciencias: unas relaciones controvertidas, Enseñanza de las Ciencias, Vol. 4, pp. 111-121.

GIL, D. y CARRASCOSA, J., 1985. Science learning as a conceptual and methodological change, European Journal of Sciencie Education, Vol. 7, pp. 231-236.

HEMPEL, C.G., 1987. Filosofia de la ciencia natural. (Alianza Ed.: Madrid).

KHUN, T.S., 1985. La revolución copernicana. (Ariel: Barcelona).

KHUN, T.S., 1987a. La estructura de las revoluciones científicas. (F.C.E.: Madrid). paralelismo con las concepciones en determinadas épocas históricas (Saltiel y Viennot 1985). Desde esta perspectiva, y teniendo en cuenta que la pedagogía actual con sus modernos aparatos de laboratorio desvirtúa muchas veces la imagen histórica de la relación entre ciencia creativa y medición (Khun 1987c), proponemos la utilización del péndulo para proceder a una verdadera reconstrucción histórica en el aula; es decir, la reproducción de razonamientos y experiencias que emplearon Galileo, Huygens, Hooke, Newton y Foucaut, a la hora de abordar cuestiones como la caída de graves, el problema histórico de la medición del tiempo, el principio de conservación de la energía mecánica, los conceptos de masa, peso y gravedad, el movimiento de los planetas, Ia importancia de los sistemas de referencia inerciales, etc. Por otro lado, dicha reconstrucción histórica podría convertirse en una valiosa oportunidad de introducir la metodología científica (Gil y Carascosa 1985) en el proceso de enseñanza/aprendizaje.

KHUN, T.S., 1987b. La conservación de la Energía como ejemplo de descubrimiento simultáneo, en La Tensión Esencial. (F.C.E.: México).

KHUN, T.S. $1987 \mathrm{c}$. La función de Ia medición en la.física moderna, en La Tensión Esencial. (F.C.E., México).

KOYRÉ, A., 1980. Estudios Galileanos. (Siglo XXI: Madrid).

KOYRÉ, A., 1983. Un experimento de medición, en Estudios de historia del pensamiento científico. (Siglo XXI: Madrid).

LOSEE, T., 1987. Introducción histórica a la filosofía de la ciencia. (Alianza Ed.: Madrid).

MASON, S.F., 1985. Historia de las Ciencias. (Alianza Ed.: Madrid).

MATTHEWS, M.R., 1990. History, Philosophy and Science Teaching: A Rapprochement, Studies in Science Education, Vol. 18, pp. 25-51.

NEWTON, I. 1987. Principios matemáticos de la filosofia natural. (Alianza Ed.: Madrid).

PAPP, D, 1945. Historia de la Física. (Espasa Calpe: Madrid).

SALTIEL, E y VIENNOT, L., 1985. ¿Qué aprendemos de las semejanzas entre las ideas históricas y el razonamiento espontáneo de los estudiantes?, Enseñanza de las Ciencias, Vol. 3, pp. 137-144.

SYMON, K.R., 1979. Mecánica. (Aguiłar: Madrid).

THUILLIER, P., 1983. Galileo y la experimentación, Mundo Científico, Vol. 3, pp. 584-597.

THUILLIER, P., 1990. De Arquimedes a Einstein. Las caras ocultas de la investigación científica. (Alianza Ed.: Madrid).

TRUESDELL, C., 1975. Ensayos de historia de la mecánica. (Tecnos: Madrid).

WOLF, C., 1889a. Introduction historique, en Mémoires sur le pendule. Collection de Mémoires relatifs a la Physique. Tome IV. (Gauthiers-Villars et fils: París).

WOLF, C., 1889b. Bibliographie du pendule, en Mémoires sur le pendule. Collection de Memoires relatifs a la Physique. Tome IV. (Gauthiers-Villars et fils: París). 